\title{
EDITORIAL
}

\section{REFLECTION ON JM ${ }^{3}$ OVER THE LAST THREe YeARS}

This journal was inaugurated in April 2002. As of the April 2005 issue, we have reached our three-year milestone. A total of 160 articles have been published. This includes papers and communications but does not include editorials by this editor or guest editors. The total number of articles multiplied by the number of authors in each article is 757 , leading to 4.73 authors per article on average. The largest number of authors in an article is 27 , spread out in four countries. The distribution of multiple-author articles is shown in Fig. I. Three authors per article are found most frequently. Not too many articles have more than eight authors. Our submission base is indeed global, because the authors are from twenty countries in four continents, as

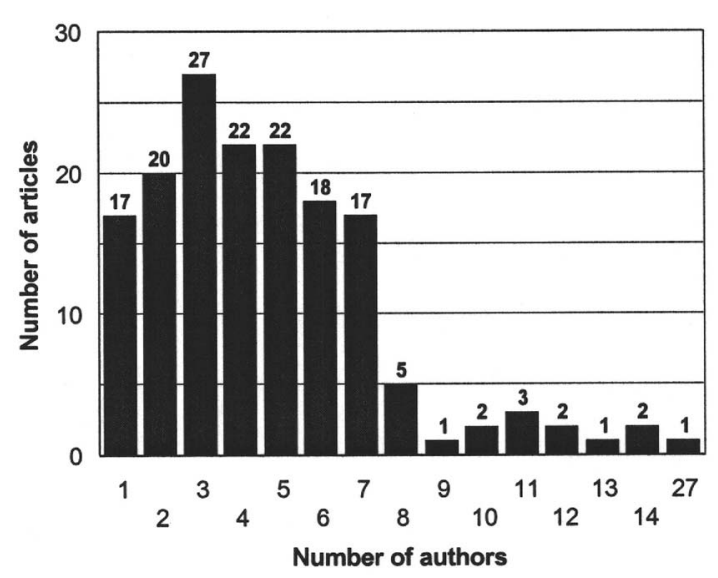

Fig. 1 Frequency of appearance of multi-authored articles.

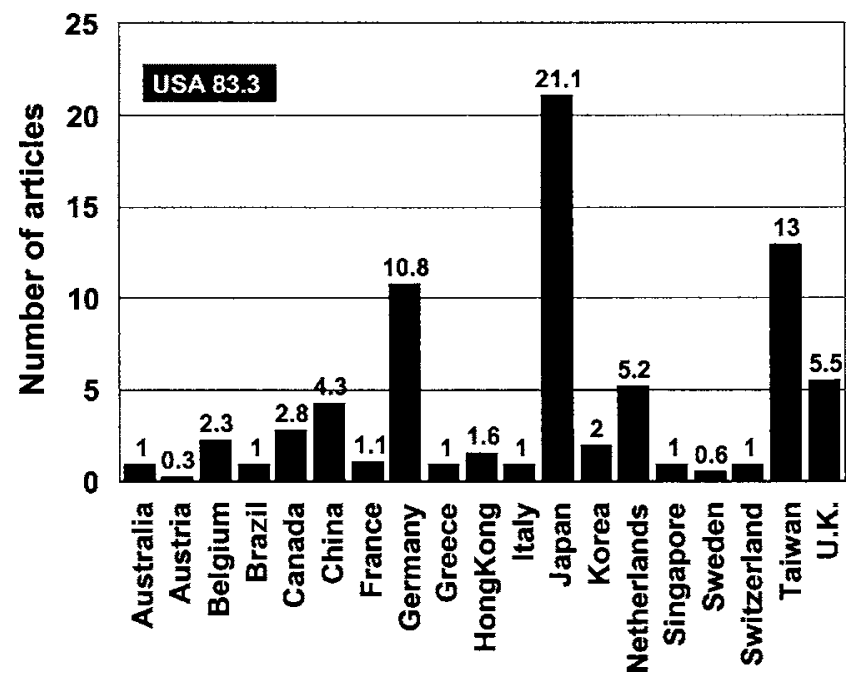

Fig. 2 Number of articles from each country. depicted in Figs. 2 and 3. Note that the number of articles per country is in fractions. This is due to multi-authored articles from many countries. For example, in the article authored by 27 people, 7 are from Austria, so their part is $7 / 27=0.259$. The papers from the US are most numerous, namely 83.3. Runners-up are Japan, Taiwan, and Germany. From their current activities, we expect more contributions from Belgium, China, France, Korea, and Sweden. In fact, I would be excited about an international competition on papers published in $\mathrm{JM}^{3}$.

The contributions are from three types of organizations: industry, universities, and research labs (see Fig. 4). The latter include ASET, IMEC, ITRE, MIT Lincoln Labs, NIST, Sandia

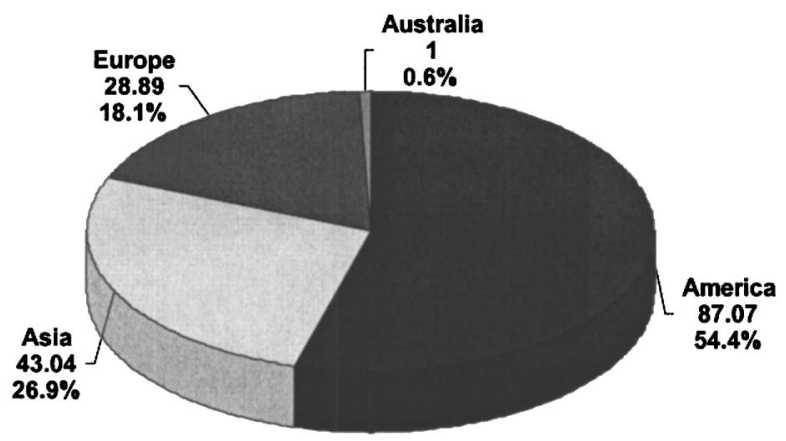

Fig. 3 Distribution of articles by continent.

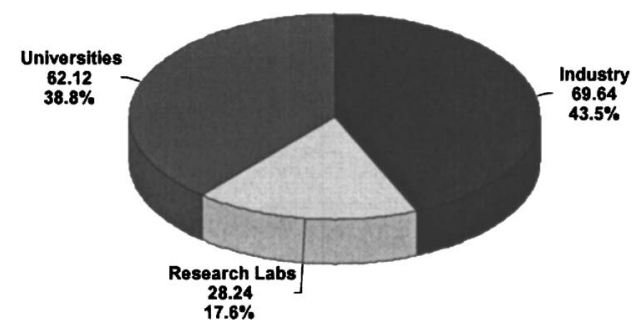

Fig. 4 Author distribution by type of organization.

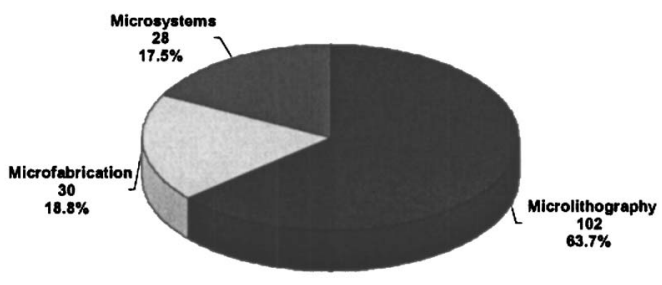

Fig. 5 Distribution by topic area. 


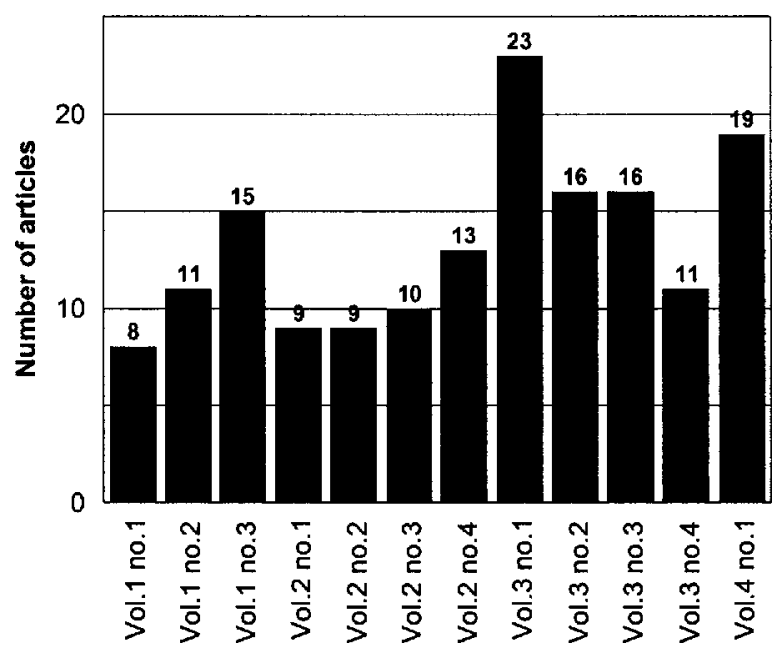

Fig. 6 Number of articles in each issue.

National Labs, Sematech, etc. I am glad to see a reasonable portion of contributions from industry and research labs. I hope they continue their enthusiasm to write. I also welcome more contributions from universities. With $\mathrm{JM}^{3}$ in the citation indexes, ongoing improvement of our reputation in quality, e-publishing, and streamlining of our review cycle, I encourage more colleagues from universities to adopt $\mathrm{JM}^{3}$ as their home journal.
The distribution in the three main areas is shown in Fig. 5. There are 102 articles on microlithography, and 30 and 28 for microfabrication and microsystems, respectively. We definitely would like to see more articles in microfabrication and microsystems. With the large number of MOEMS papers in the upcoming issue, we would like to bring the message across that $\mathrm{JM}^{3}$ is also a home journal for MEMS and MOEMS works. The synergy of these areas fosters a common bond among our readers; workers in one area often can benefit from the work of the other areas. MEMS and MOEMS authors have an audience larger than just those in these two fields. The readers can enjoy microlithography literature in addition to these two fields.

The growth of $\mathrm{JM}^{3}$ is shown in Fig. 6. It started with eight articles in the inaugural issue, and quickly grew to 15 articles, averaging II articles per year in 2002. In 2003, the average was 10; in 2004, 17. The issue with the largest number of articles is Vol. 3, No. I, which includes 13 articles in the immersion special section. We are looking forward to sustaining the 2004 average in 2005 , if not exceeding it.

Happy reading!

\section{Burn J. Lin Editor-in-Chief}

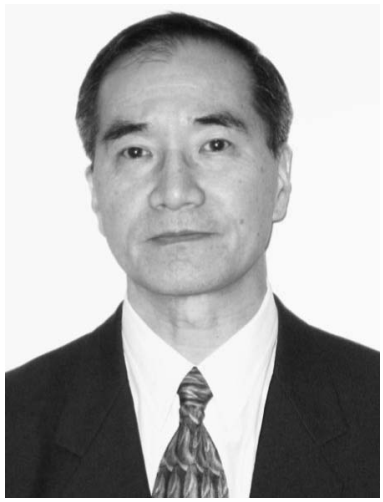

